\title{
Solar System Science with Robotic Telescopes
}

\author{
T. A. Lister \\ Las Cumbres Observatory Global Telescope Network (LCOGT), Goleta, CA 93117, USA \\ email: tlister@lcogt.net
}

\begin{abstract}
An increasing number of sky surveys is already on-line or soon will be, leading to a large boost in the detection of Solar System objects of all types. For Near-Earth Objects (NEOs) that could potentially hit the Earth, timely follow-up is essential. I describe the development of an automated system which responds to new detections of NEOs from Pan-STARRS and automatically observes them with the LCOGT telescopes. I present results from the first few months of operation, and plans for the future with the 6-site, 40-telescope global LCOGT Network.
\end{abstract}

Keywords. minor planets, asteroids, astrometry, celestial mechanics, telescopes

\section{Introduction}

Scientific interest in asteroids is motivated by their status as remnants from the solarsystem formation process. Their study can provide insights into the assembly of terrestrial planets and to planetary formation mechanisms in general. Near-Earth Objects (NEOs) are the closest neighbours of the Earth-Moon system, and research into them is important not only for Solar System science but also for understanding and protecting human society from potential impact hazards. NEOs originated in collisions between bodies in the main asteroid belt, and have found their way into near-Earth space via complex dynamical interactions. This transport of material from the main belt into the inner Solar System has shaped the histories of the terrestrial planets.

Moving objects such as asteroids, which have typical rates of motion between about $1^{\prime \prime}-10^{\prime \prime} /$ min for NEOs and $2^{\prime \prime}-3^{\prime \prime} / \mathrm{hr}$ for Kuiper Belt Objects, add an extra complexity to the population of transients being discovered in time-domain surveys. New sky surveys such as Pan-STARRS (PS1; Kaiser 2004) and the Palomar Transient Factory (PTF; Law et al. 2009) which have recently started operating have the capability of discovering large numbers of new solar-system objects and thus to increase greatly our understanding of the solar-system formation and evolution processes. They will be joined by upcoming surveys such as LSST (Ivezić et al. 2008) and SkyMapper (Keller et al. 2007). There is a coupled growth in the number of robotic telescopes which can respond automatically and follow up new detections from those sky surveys.

PS1 uses a 1.8-m telescope coupled with a 1.4-Gigapixel CCD camera, and images a 7-deg. ${ }^{2}$ field with a variety of survey cadences which in turn yield NEO detections. The PS1 cadence is not enough by itself to confirm newly-detected NEOs; that is where the follow-up network comes in. In order to cope with the large number of NEO candidates that PS1 produces, I have developed an automated system to retrieve new PS1 NEOs, compute their orbits, plan observations and automatically schedule them for follow-up on the robotic telescopes of the LCOGT Network (Shporer et al. 2010; also p. 408). The current follow-up telescope are the two 2-m Faulkes Telescopes (FTN: Maui, Hawaii; FTS: Siding Spring, Australia) and the 0.8-m Sedgwick telescope (Sedgwick Preserve, CA). 


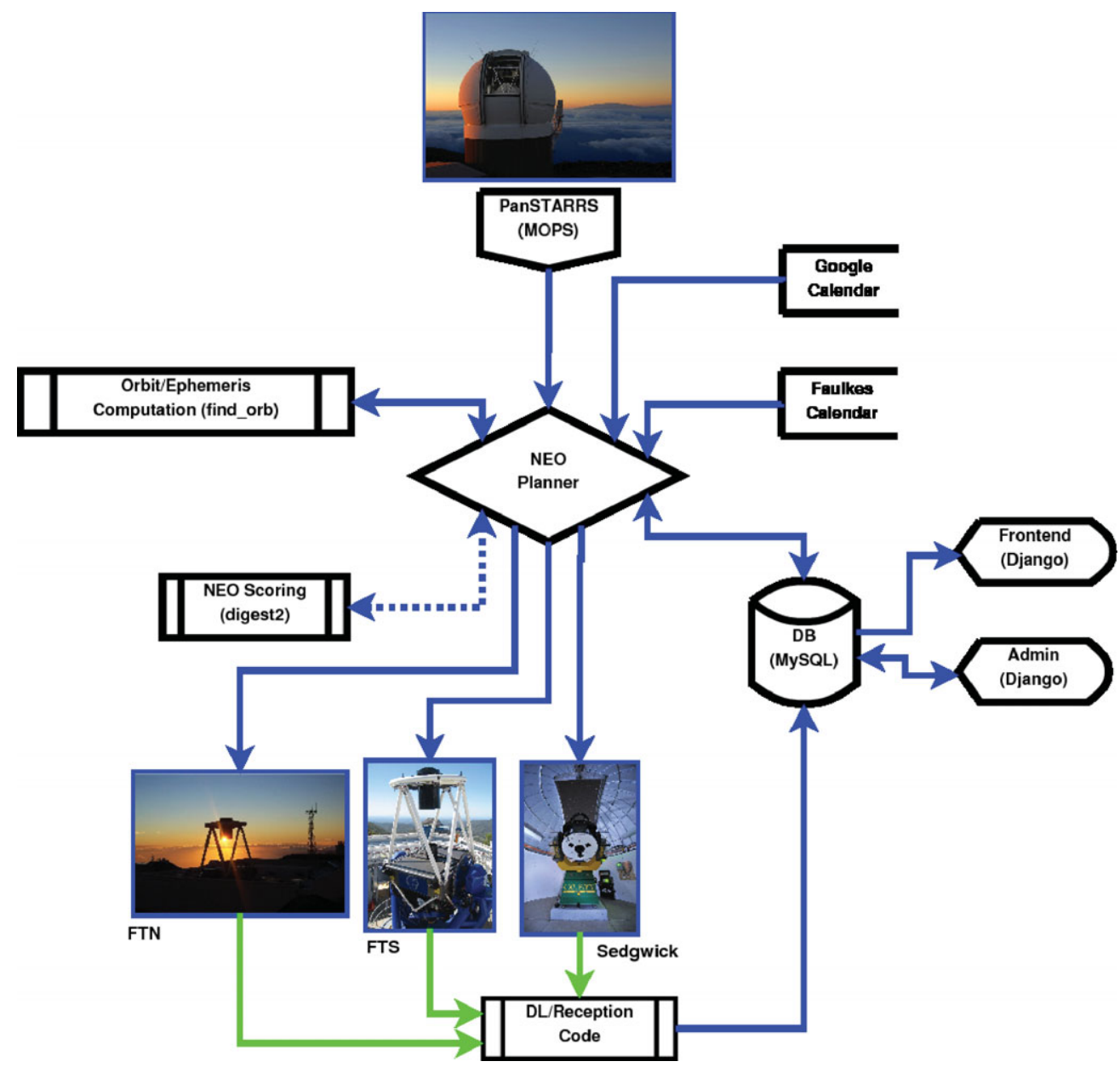

Figure 1. Overall layout of the automated follow-up system.

\section{Automated Follow-Up Planning}

The overall layout of the automated follow-up systems is shown in Fig. 1 above. The planning process proceeds by:

- retrieving new PS1 NEO candidates from the Moving Object Processing System,

- calculating new orbits and ephemerides for the selected candidates,

- computing the subset of an object's ephemeris while it is dark, the object is $>30^{\circ}$ altitude and telescope is unbooked (valid slots are scored based on the target altitude and distance from the Moon),

- estimating exposure times from the object's rate of motion and the pixel scale of the particular detector, balanced by the need for at least 3 exposures within the chosen block length,

- submitting observations to the telescope and the recording the details in a Django database (DB),

- fetching frames of successfully observed targets and recording them in the DB. 
The code makes extensive use of Open Source software: the main code is Python, with Starlink SLALIB (Wallace 1994) for astronomical calculations. The Google Data API (for Sedgwick) and MySQL (for the two Faulkes telescopes) are used for fetching telescope bookings. The writing of the front-end uses the Django web framework, with a MySQL back-end.

\section{The LCOGT Network}

Over the next few years the LCOGT Network will undergo a major expansion from the existing three telescopes to $121-\mathrm{m}$ telescopes and $240.4-\mathrm{m}$ ones. They will be deployed over six sites; those in the Southern Ring (CTIO, Chile; SAAO, South Africa; Siding Spring, Australia) will be completed first, followed by the northern sites (McDonald Obs., TX; Tenerife, Canary Islands; Asian site TBD). All the 1-m telescopes, which are most suitable for NEO follow-up, will be equipped with CCD cameras and a standardized set of 21 filters (including Sloan and Bessell sets).

A worldwide network of robotic telescopes capable of continuous coverage and greater availability, when combined with dynamic scheduling, will create a very powerful resource for NEO follow-up.

\section{Results and Future Work}

The automated follow-up system has been in operation from early April 2011, and has successfully queued and observed $\sim 200$ objects. Of those, a high percentage $(\sim 75 \%)$ of groups that were actually observed were successfully reported to the Minor Planet Center. During 2011 those observations resulted in over 60 Minor Planet Electronic Circulars announcing new objects of interest.

Follow-up with LCOGT telescopes has given rise to the confirmation of 65 new objects from PS1 and other surveys. Those objects include 27 NEOs, 4 comets, 2 Centaurs and 32 other objects (primarily Main-Belt asteroids). In the course of the survey follow-up I have discovered 7 new Main-Belt objects and 2 new Jupiter Trojans.

Observations with the $0.8-\mathrm{m}$ Sedgwick telescope have recovered objects as faint as $V \sim 21$. The 1-m network therefore has substantial potential for NEO follow-up as the instruments will have greater collecting area, better CCD cameras (with higher QE), and will be situated at better-quality sites.

\section{References}

Ivezić, Z. et al. 2008, arXiv:0805.2366

Kaiser, N. 2004, SPIE Proceedings, 5489, 11

Keller, S., et al. 2007, PASA, 24, 1

Law, N., et al. 2009, PASP, 121, 1395

Shporer, A., et al. 2010, IAUS 276, arXiv:1011.6394

Wallace, P. T. 1994, in: D. R. Crabtree, R. J. Hanisch and J. Barnes (eds.), ADASS III, ASPC, 61 (San Francisco: ASP), p. 481 